Pathologe 2010 · [Suppl 2] 31:177-182

DOI 10.1007/s00292-010-1331-0

Online publiziert: 28. Juli 2010

(c) Springer-Verlag 2010

T. Kalinski · A. Roessner

Institut für Pathologie, Otto-von-Guericke-Universität, Magdeburg

\title{
Angiogenese in Knorpeltumoren
}

könnten. Darüber hinaus könnte die Regulation der Angiogenese möglicherweise therapeutisch von Bedeutung sein.

\section{Grundlagen der Angiogenese}

Für Tumorwachstum und -progression ist eine adäquate Versorgung mit Nährstoffen und Sauerstoff über das Blut erforderlich. Daher spielt die Angiogenese in neoplastischem Gewebe eine wesentliche Rolle [3]. Auf molekularer Ebene liegt der Angiogenese ein Gleichgewicht zwischen pro- und antiangiogenen Faktoren zugrunde, deren Wirkung bei Verschieben des Gleichgewichts auf die Seite der proangiogenen Faktoren mit dem Einschalten eines angiogenen Schalters vergleichbar ist [5]. Ein Maß für die Angiogenese ist die Mikrogefäßdichte (MVD, „microvessel density“), die in vielen Tumoren mit einer schlechten Prognose assoziiert ist [6].

Der Schlüsselfaktor der Angiogenese ist VEGF-A („vascular endothelial growth factor A“). Die Wirkung von VEGF-A wird über die VEGF-Rezeptoren- (VEGFR-) 1 und 2 auf Endothelzellen vermittelt, wobei über VEGFR-2 die wesentlichen Effekte, wie Steigerung der Gefäßpermeabilität, Überleben, Proliferation, Invasion und Migration von Endothelzellen vermittelt werden [26]. Eine schlechte Prognose verbunden mit einer Überexpression von VEGF-A konnte in vielen humanen Tumoren nachgewiesen werden, z. B. in Kolon-, Pankreas- und Magenkarzinomen, in Bronchial-, Mamma- und Prostatakarzinomen sowie in Melanomen [6].

Von VEGF-A existieren 6 Splice-Varianten, aus denen Proteine unterschiedlicher Länge hervorgehen (VEGF-A $A_{121}$, VEGF-A $A_{145}$, VEGF-A $A_{165}$, VEGF-A $A_{183}$,
VEGF- $\mathrm{A}_{189}$ und VEGF- $\mathrm{A}_{209}$ ), die unterschiedliche biochemische Eigenschaften besitzen, welche Einfluss auf deren Bioverfügbarkeit haben [23]. Die längeren Varianten sind in der Extrazellulärmatrix gebunden und werden erst bei Freisetzung aktiv, wohingegen die kürzeren Varianten frei löslich sind. VEGF- $A_{165}$ ist die prädominante Isoform in zahlreichen Tumoren, wobei andere VEGF-A-Isoformen teilweise gewebsspezifisch sind [6].

ADAMTS$_{1}$ („a disintegrin and metalloproteinase with thrombospondin motifs 1) ist ein Antagonist von VEGF-A, der durch Bindung von VEGF-A das angiogene ,signaling “ über VEGFR-2 auf Endothelzellen verhindert [7]. Die Bindung von VEGF-A ist auf die 3 ThrombospondinMotive des ADAMTS1-Proteins zurückzuführen, wobei für ADAMTS1 eine wesentliche höhere antiangiogene Wirkung als für Thrombospondin im gleichen molaren Verhältnis beschrieben wurde [17]. Darüber hinaus besitzt ADAMTS1 eine Metalloproteinaseaktivität und kann z. B. Aggrecan, einen Bestandteil der Knorpelmatrix, spalten, wodurch ADAMTS1 auch bei pathologischen Prozessen mit Umbau kartilaginärer Matrix, wie bei entzündlichen Gelenkerkrankungen oder Tumoren, eine Rolle spielt [18].

Neben VEGF-A sind zahlreiche weitere proangiogene Faktoren an der Angiogenese beteiligt. Speziell gehören dazu die Angiopoietine- (Ang-) 1 und 2, deren Wirkung über spezifische Tie-2-Rezeptoren auf Endothelzellen vermittelt wird, wobei Ang-1 als Agonist und Ang-2 auch als Antagonist wirken kann [8]. Angiopoietine haben eine Funktion als Überlebensfaktor für Endothelzellen, wobei Ang-1 zusätzlich Einfluss auf Migration 

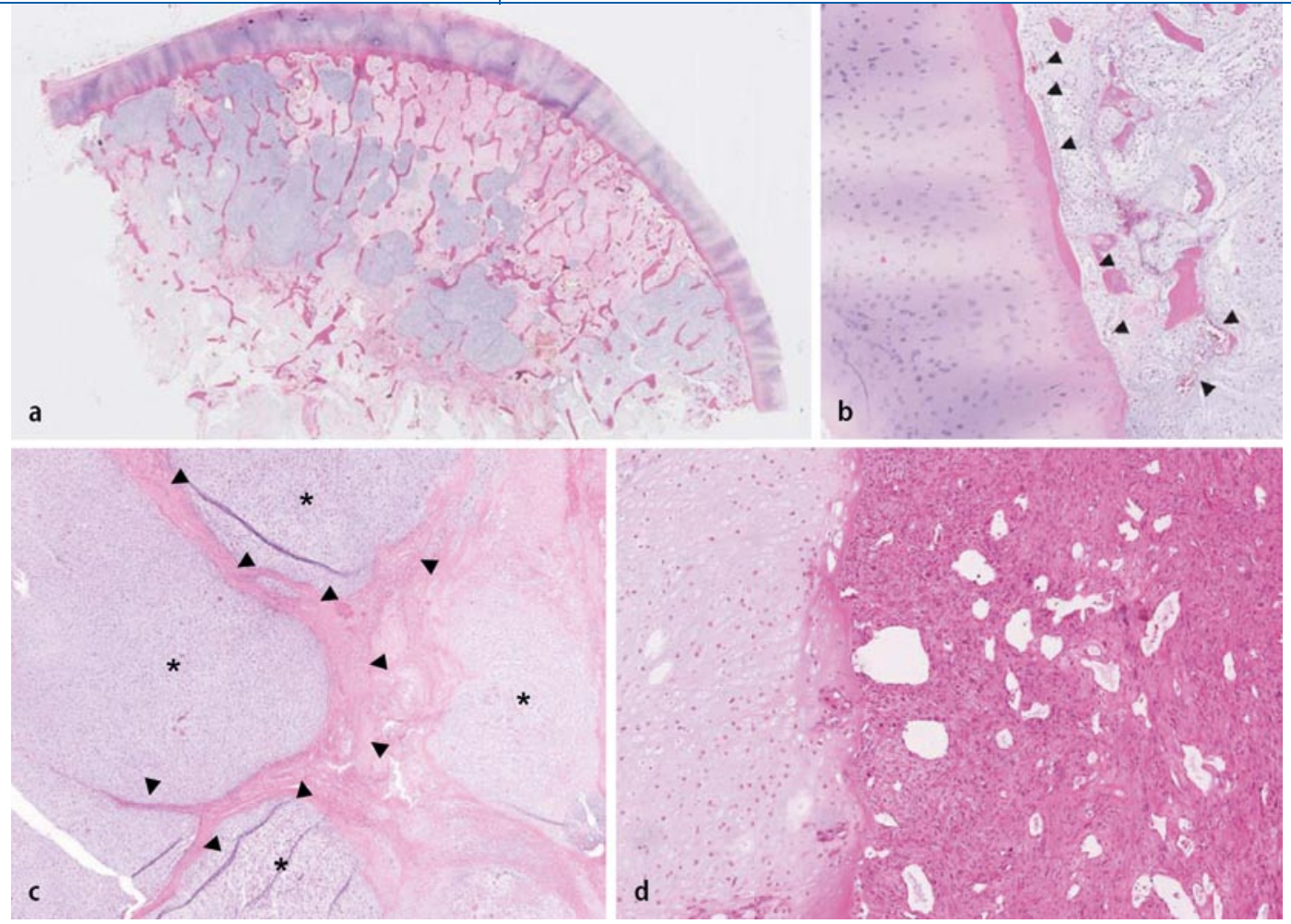

Abb. $1 \varangle$ Morphologie (virtuelle Mikroskopie). a Chondrosarkom Grad II in einem Humeruskopf. b (Vergrößerung von a) Avaskuläres Gelenkknorpelgewebe neben Chondrosarkom mit Blutgefäßen (Pfeilspitzen). c Chondrosarkom Grad II mit fibrovaskulären Septen (Pfeilspitzen) und kartilaginären Tumorknoten (Sternchen).

d Dedifferenziertes Chondrosarkom mit gut differenzierter kartilaginärer Komponente (links) und hochmaligner dedifferenzierter Komponente (rechts), einem malignen fibrösen Histiozytom entsprechend

Gefäßmuster in Knorpeltumoren

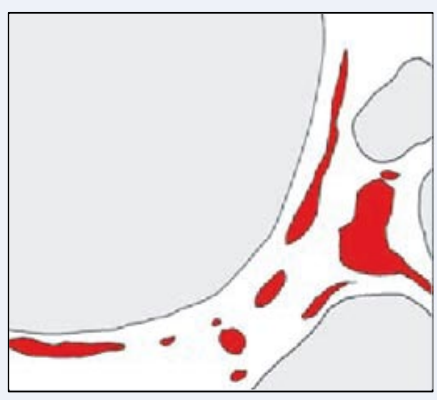

fibrovaskuläre

Septen:

Enchondrom, Chondrosarkom Grad I,II,III

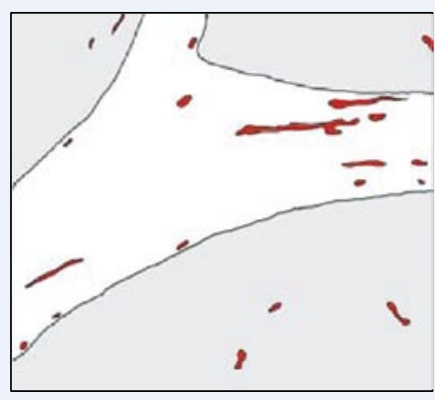

fibrovaskuläre

Septen + intrakartilaginäre Gefäße:

Chondrosarkom Grad II,III

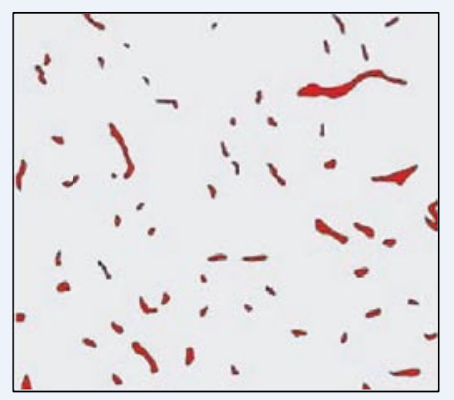

diffus

perikartilaginär:

dedifferenziertes

Chondrosarkom
Abb. $2 \varangle$ Morphologische Muster der Angiogenese in Knorpeltumoren und Adhäsion von Endothelzellen sowie auf die Gefäßreifung und -stabilisierung durch die Rekrutierung von Perizyten bzw. glattmuskulären Zellen hat, wohingegen Ang-2 eine destabilisierende, entgegengesetze Wirkung auf Perizyten besitzt, welche einer Gefäßsprossung vorangeht [20]. In vielen Tumoren konnte eine Überexpression von Ang-2 nachgewiesen werden, z. B. in nichtkleinzelligen Bronchialkarzinomen mit prognostischer Relevanz [21]. Für Ang-1 ist dagegen eher eine gleichbleibende, höhere oder niederige Expression beschrieben worden [21].

$\mathrm{Zu}$ weiteren Faktoren mit proangiogener Wirkung zählen u. a.: PDGF, TGF- $\beta$,
FGF, HGF, MCP-1, einige Integrine, Ephrine, Metalloproteinasen und Plasminogenaktivatoren, PAI-1, NOS und COX-2. Faktoren mit antiangiogener Wirkung sind u. a. Thrombospondin-1 und -2, Angiostatin, Endostatin, Vasostatin, Plättchenfaktor 4, TIMPs, Meth1 und Meth2, Interferon- (IFN-) $\alpha, \beta$ und $\gamma$, Interleukin- (IL-) 4, 12 und 18 [3]. Abgesehen davon kann es noch andere, z. T. auch unbekannte Faktoren geben, die eine Rolle in der Angiogenese spielen.

Ein morphologischer Indikator für die Gefäßreifung ist die Rekrutierung von Perizyten bzw. glattmuskulären Zellen, die durch Interaktion einen stabilisierenden
Effekt auf neugebildete Blutgefäße ausüben, z. B. durch Ang-1/Tie-2-Signaling [22]. Der Grad der Perizytenrekrutierung lässt sich als Index (MPI, „microvessel pericyte coverage index") in verschiedenen Tumoren bestimmen. Die Perizytenrekrutierung kann zwar auf eine Gefäßreifung hinweisen, stellt aber keinen Endzustand da, da sie reversibel ist, z. B. durch Ang-2.

Die angiogene Aktivität in Tumoren kann an der Proliferation der Endothelzellen in den Kapillaren, gemessen als kapillärer Proliferationsindex (PCI, ,proliferating capillary index"), abgelesen werden. Auch bezüglich des PCI wurden zu- 
vor Unterschiede in verschiedenen Tumoren beschrieben [4]. Abgesehen von der Quantität der Angiogenese (MVD), ist mit MPI und PCI danach auch eine qualitative Aussage zur Angiogenese möglich.

\section{Ausprägung der Angiogenese in kartilaginären Tumoren}

Im Gegensatz zu anderen Tumoren weist die Angiogenese in kartilaginären Tumoren Besonderheiten auf. Dabei sind die Blutgefäße in Enchondromen und konventionellen Chondrosarkomen typischerweise in fibrovaskulären Septen zwischen den knotigen Tumorformationen angeordnet (• Abb. 1 c).

In einigen höhergradigen Chondrosarkomen lassen sich fokal zusätzlich intrakartilaginäre Blutgefäße nachweisen $[1,10]$. In dedifferenzierten Chondrosarkomen ist dagegen eine diffuse, perikartilaginäre Verteilung von Blutgefäßen in der hochmalignen Tumorkomponente mit Aussparung der gut abgrenzbaren kartilaginären Komponente zu erkennen. Die Ursache für dieses Phänomen kann vermutlich auf die antiangiogene Wirkung der kartilaginären Matrix durch spezielle knorpelassoziierte Proteine, wie z. B. Chondromodulin, Troponin I und Thrombospondin-1 zurückgeführt werden.

Das Auftreten von intrakartilaginären Blutgefäßen in einigen konventionellen Chondrosarkomen sowie auch bei der chronischen Polyarthritis beweist, dass eine Angiogenese auch im Knorpelgewebe bzw. in Knorpeltumoren trotz der antiangiogenen Wirkung der Knorpelmatrix grundsätzlich möglich ist. In • Abb. 2 sind die distinkten Gefäßmuster von Knorpeltumoren schematisch dargestellt.

Wesentliche Unterschiede bei der Angionese in Enchondromen, konventionellen Chondrosarkomen und dedifferenzierten Chondrosarkomen lassen sich zunächst in quantitativer Hinsicht erkennen. So lässt sich in dedifferenzierten Chondrosarkomen eine signifikant höhere Mikrogefäßdichte nachweisen, im Durchschnitt um das 4- bis 5-Fache höher als in Enchondromen und konventionellen Chondrosarkomen [11, 12]. Zwischen Enchondromen und konventionellen Chondrosarkomen sind die Unterschiede ge- ringer und nicht signifikant, mit etwas höherer MVD in höhergradigen Chondrosarkomen. Die in Enchondromen im Vergleich zu Chondrosarkomen Grad I leicht erhöhte MVD ist ein Hinweis auf die unterschiedliche Natur dieser Entitäten mit einer höheren proliferativen Aktivität insbesondere in Enchondromen aus den kleinen Finger- oder Zehenknochen [11].

Ähnlich wie Kolon- und Mammakarzinome [4] zeigen Knorpeltumoren einen überwiegend reifen angiogenen Phänotyp (- Abb. 3 a-f) mit hohem MPI in Enchondromen und konventionellen Chondrosarkomen $(>70 \%)$, in dedifferenzierten Chondrosarkomen überraschenderweise sogar mit einem signifikant noch höheren MPI (>90\%) als Ausdruck der unterschiedlichen Natur dieser Entitäten [12]. Eine schlechtere Prognose bei niedrigem MPI wie bei Kolonkarzinomen [27] lässt sich bei Chondrosarkomen mit Blick auf dedifferenzierte Chondrosarkome nicht erkennen, eine spezielle Verteilung der Gefäße mit reifem Phänotyp ebenfalls nicht, wobei Perizyten interessanterweise auch um intrakartilaginäre Gefäße nachzuweisen sind [12], die zweifelsfrei der tumorassoziierten Gefäßbildung zugeordnet werden können. Die Rekrutierung von Perizyten stellt offenbar auch in Chondrosarkomen ein frühes Ereignis bei der Gefäßsprossung und Gefäßbildung dar [16].

Hinsichtlich der Gefäßproliferation (- Abb. 4 a-d) kann in höhergradigen konventionellen Chondrosarkomen ein signifikant höherer PCI als in Enchondromen und Chondrosarkomen Grad I beobachtet werden, wohingegen der PCI in dedifferenzierten Chondrosarkomen nicht höher ist als in Enchondromen und Chondrosarkomen Grad I [12]. Die Gefäßproliferation korreliert damit gut mit der Expression von VEGF-A in kartilaginären Tumoren [11]. Insgesamt lässt sich damit zwar ein differenzielles Bild der Angiogenese in Knorpeltumoren erkennen, eine differenzialdiagnostische Hilfe speziell bei Enchondromen, bzw. Chondrosarkomen Grad I lässt sich daraus jedoch nicht ableiten.
Pathologe 2010 · [Suppl 2] 31:177-182

DOI 10.1007/s00292-010-1331-0

C) Springer-Verlag 2010

T. Kalinski · A. Roessner

Angiogenese in

Knorpeltumoren

Zusammenfassung

Im Gegensatz zu normalem Knorpelgewebe, welches avaskulär ist, ist die Angiogenese ein wesentliches Merkmal von Knorpeltumoren. In dieser Übersicht stellen wir die Prinzipien der Angiogenese im Hinblick auf neueste Befunde zu differenziellen morphologischen und molekularen Aspekten der Angiogenese in Knorpeltumoren, einschließlich Enchondromen, konventionellen Chondrosarkomen und dedifferenzierten Chondrosarkomen, vor. Ferner beschreiben wir den Einfluss von Hypoxie und Interleukin- $1 \beta$ auf das angiogene „signaling" in Chondrosarkomzellen.

Schlüsselwörter

Angiogenese - Knorpeltumoren - Zellkultur . Interleukin-1 $\beta$. Hypoxie

\section{Angiogenesis in cartilage tumors}

Abstract

In contrast to normal cartilage, which is avascular, angiogenesis is characteristic of cartilage tumors. In this review, we outline the basic principles of angiogenesis with regard to recent findings on differential morphological and molecular aspects of angiogenesis in cartilage tumors, including enchondromas, conventional chondrosarcomas and dedifferentiated chondrosarcomas. Furthermore, we describe the effects of hypoxia and interleukin$1 \beta$ on angiogenic signaling in chondrosarcoma cells.

Keywords

Angiogenesis - Cartilage tumors - Cell culture . Interleukin-1 $\beta \cdot$ Hypoxia 


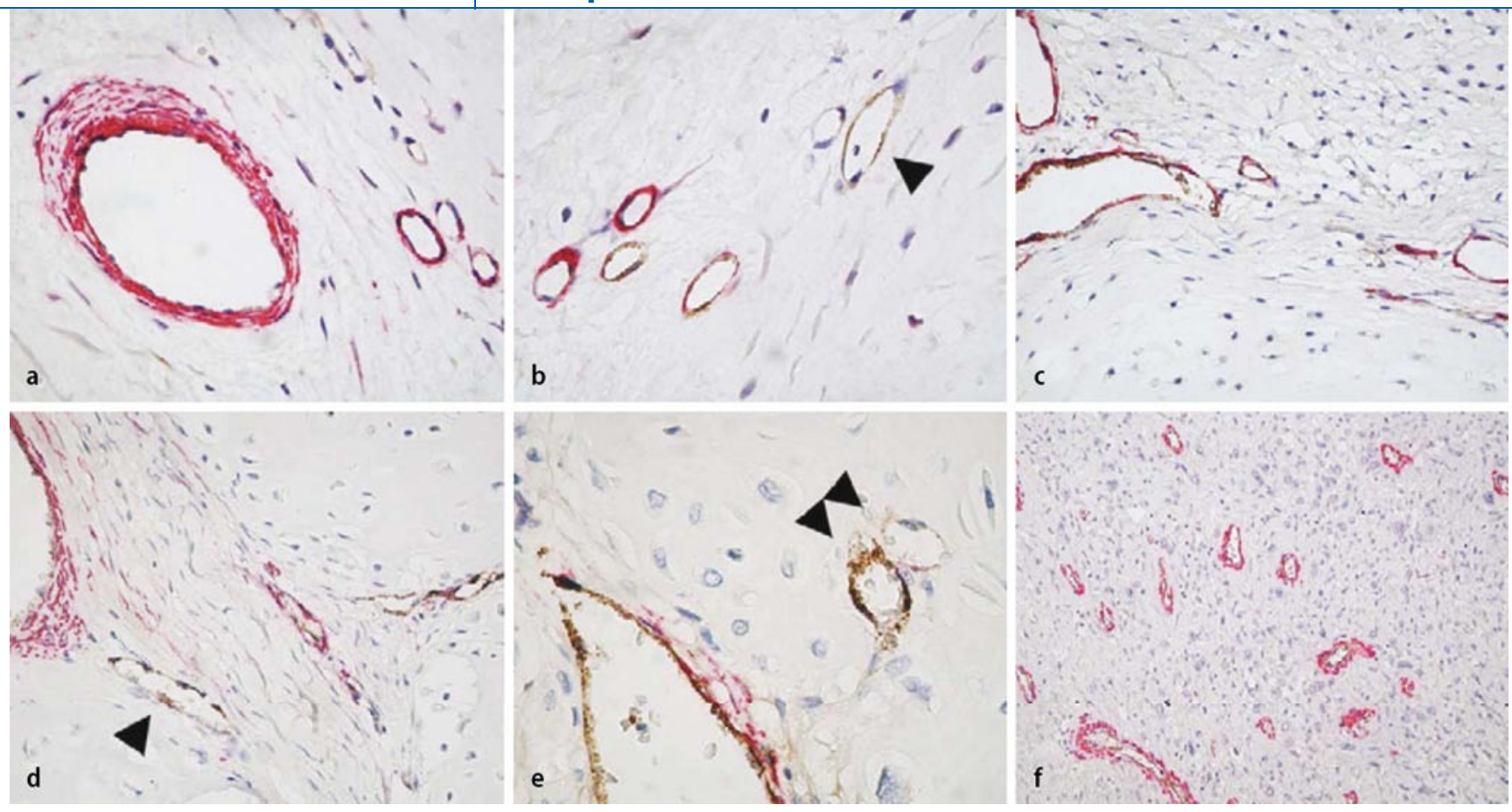

Abb. $3 \Delta$ MPI in Knorpeltumoren. a Fibröses Septum mit Einschluss eines größeren Blutgefäßes mit multiplen Schichten von Perizyten bzw. glatten Muskelzellen in einem Enchondrom. b Blutgefäße, teilweise ohne Perizyten (Pfeilspitze) in einem Enchondrom. c Fibröses Septum mit Blutgefäßen in einem Chondrosarkom Grad I. d Fibröses Septum mit Blutgefäßen, fokal ohne Perizyten (Pfeilspitze), in einem Chondrosarkom Grad II. e Intrakartilaginäre Blutgefäße (Pfeilspitzen), hier ohne Perizyten, in einem Chondrosarkom Grad II. f Diffus verteilte Blutgefäße mit Perizyten in einem dedifferenzierten Chondrosarkom. Doppelimmunhistochemie von-Willebrand-Faktor (braun)/glattmuskuläres Aktin (rot). (Mod. nach [12])

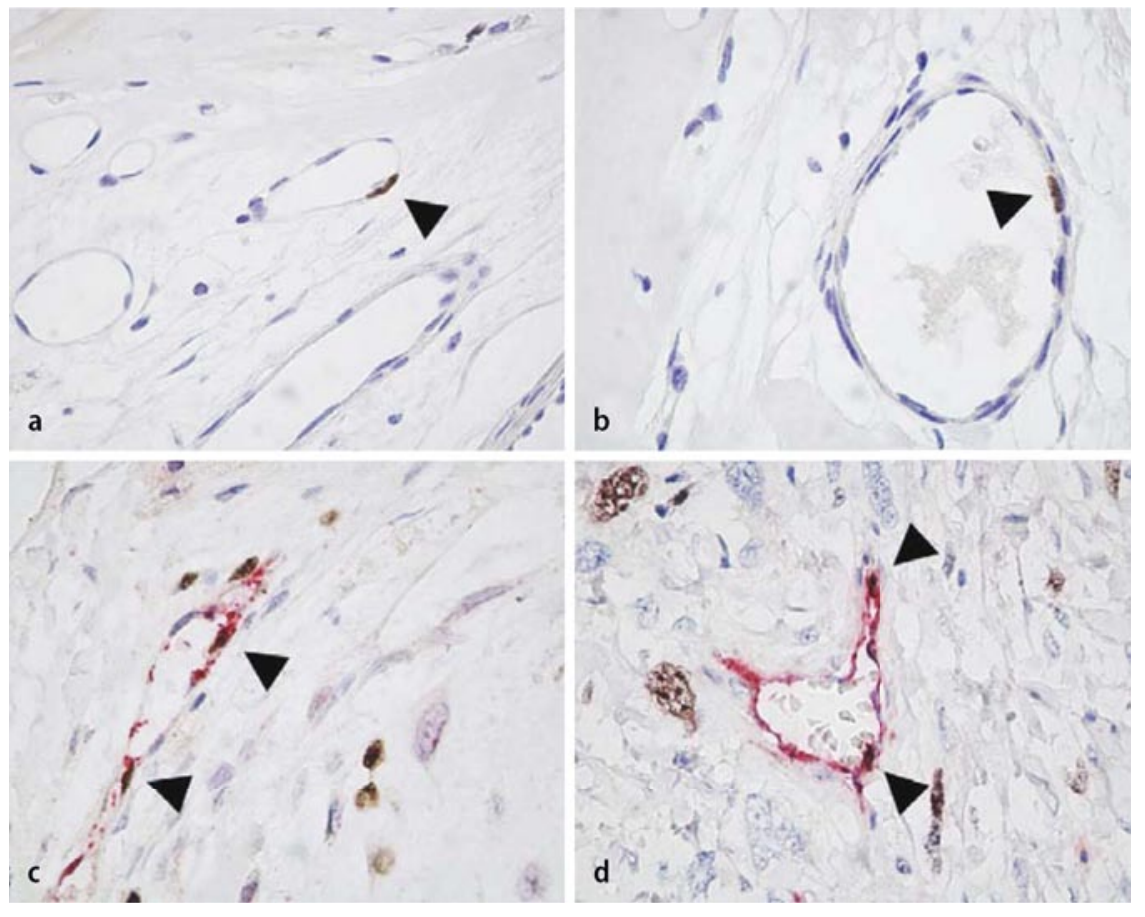

Abb. 4 A PCl in Knorpeltumoren. a MIB-1-Positivität einer Endothelzelle (Pfeilspitze) in einem Enchondrom. b Ähnlicher Befund (Pfeilspitze) in einem Chondrosarkom Grad I. c MIB-1-Positivität (Pfeilspitzen) von Endothelzellen in einem Chondrosarkom Grad II. d Ähnlicher Befund (Pfeilspitzen) in einem dedifferenzierten Chondrosarkom. (Aus [12], mit freundlicher Genehmigung von Elsevier)

\section{Expression von Angiogenesefaktoren in kartilaginären Tumoren}

Die Expression von VEGF-A in Knorpeltumoren ist transkriptionell reguliert und weist sowohl in Enchondromen als auch in konventionellen und dedifferenzierten Chondrosarkomen jeweils ein typisches Muster von 4 Isoformen (VEGF- $A_{121}$, VEGF- $_{145}$, VEGF- $A_{165}$ und VEGF- $A_{189}$ ) auf, das auch in normalem Korpelgewebe nachzuweisen ist [11]. Eine Modulation der VEGF-A-Isoformen in den verschiedenen Knorpeltumoren besteht nicht. Die mitogene Potenz der VEGF-A-Isoformen ist bei den längeren Isoformen wie VEGF$A_{165}$ stärker ausgeprägt [14], wobei angenommen werden kann, dass die Gefäßproliferation in Knorpeltumoren allein durch das Maß der VEGF-A-Expression beeinflusst wird. Dabei lässt sich im Vergleich zu Enchondromen, Chondrosarkomen Grad I und dedifferenzierten Chondrosarkomen eine signifikant höhere VEGFA-Expression in höhergradigen konventionellen Chondrosarkomen nachweisen, wodurch die unterschiedliche Natur der 
Entitäten noch einmal deutlich wird. Die höhere Gefäßdichte in dedifferenzierten Chondrosarkomen bei gleichzeitig niedrigerer VEGF-A-Expression kann offenbar auf den fehlenden hemmenden Einfluss durch die kartilaginäre Matrix im dedifferenzierten Tumoranteil zurückgeführt werden.

Mit Blick auf den reifen angiogenen Phänotyp erscheint die Expression von Ang-1 und die nur in Einzelfällen nachweisbare Expression von Ang-2 in Knorpeltumoren nicht überraschend [11], wobei sich nicht nur aufgrund der Ang-1-Expression in normalem avaskulärem Knorpelgewebe auch die Frage stellt, ob Ang-1 außer seiner Rolle in der Perizytenrekrutierung und Gefäßstabilisierung möglicherweise noch weitere Eigenschaften besitzen könnte [20]. Zunächst noch unbestimmt ist die Rolle von Ang-2 bei der Entstehung intrakartilaginärer Blutgefäße in höhergradigen Chondrosarkomen [11].

Die Expression des VEGF-A-Antagonisten ADAMTS1 ist im Gegensatz zu an- deren Tumoren, wie z. B. Mammakarzinomen [17], in Chondrosarkomen nicht herunterreguliert, und es besteht keine Korrelation mit der Expression von VEGF-A [10]. Anders als bei der Expression von VEGF-A lassen sich auch keine Unterschiede in der ADAMTS1-Expression in konventionellen und dedifferenzierten Chondrosarkomen nachweisen. Auch wenn die ADAMTS1-Expression in Enchondromen niedriger ist als in Chondrosarkomen [10], ist auch hiermit eine sichere differenzialdiagnostische Abgrenzung gegenüber Chondrosarkomen Grad I nicht möglich.

\section{Regulation angiogener Faktoren im Zellkulturmodell}

Bei der Regulation der Angiogenese spielen 2 Faktoren eine besondere Rolle:

- Hypoxie und

- IL-1 $\beta$.

Der wichtigste Mediator der Hypoxie ist HIF-1 („hypoxia inducible factor $1^{\text {“) }}$, ein
Heterodimer aus dem unter Hypoxie stabilisierten HIF-1 $\alpha$ und HIF-1 $\beta$, der ein Transkriptionsfaktor für zahlreiche Hypoxie-regulierte Gene ist, darunter VEGF- $A$ [24]. Die Tumorhypoxie ist in zahlreichen Tumoren mit einer schlechteren Prognose assoziiert worden, z. B. in Zervixkarzinomen [24]. Obwohl Knorpelgewebe und Knorpeltumoren wegen des Sauerstoffdiffusionslimits in der Knorpelmatrix als hypoxisch gelten kann, sind Knorpelzellen offenbar dennoch Hypoxie-sensibel und reagieren auf Hypoxie mit der Regulation von HIF-1 und VEGF-A [15, 19]. In Chondrosarkomzellen [9] konnten wir unter zunehmender Hypoxie eine Hochregulation der Expression von VEGF-A bei gleichbleibender Expression von ADAMTS1 nachweisen, was auf eine VEGF-A-unabhängige Regulation von ADAMTS1 hinweist [10].

Die Wirkung von IL-1 $\beta$ wird dagegen über den IL-1-Rezeptor vermittelt und führt zur Aktivierung der Mitogenaktivierten Protein- (MAP-) Kinase, der Phosphatidyl-Inositol-3- ( $\left.\mathrm{PI}_{3}-\right)$ Kinase so-

\section{Hier steht eine Anzeige.}

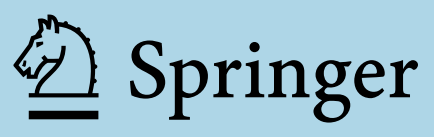


wie des nukleären Faktors kappa B (NF$\kappa \mathrm{B})$ und damit $u$. a. auch zur Regulation von VEGF-A [13]. IL-1 $\beta$ kann daher als wesentlicher Faktor bei der Tumorangiogenese angesehen werden [25].

In Chondrosarkomzellen konnten wir bei Stimulation mit IL-1 $\beta$ eine Hochregulation von VEGF-A bei gleichzeitiger Herunterregulation von ADAMTS1 nachweisen [10]. Möglicherweise hat IL-1 $\beta$ damit einen größeren Einfluss auf die Angiogenese in Chondrosarkomzellen als Hypoxie.

Insgesamt zeigen die Ergebnisse, dass angiogenes Signaling im Zellkulturmodell grundsätzlich beeinflusst werden kann, wobei therapeutisch sowohl eine positive als auch eine negative Regulation denkbar wäre. Im Hinblick auf eine antiangiogene Therapie konnten wir mittlerweile zeigen, dass durch die Substanz Curcumin eine Hemmung des IL-1 $\beta$-Signalwegs in Chondrosarkomzellen und damit auch eine Hemmung der VEGF-A-Expression möglich ist. Die Ergebnisse hierzu werden in Kürze veröffentlicht. Ob eine Regulation des angiogenen Signalings in Chondrosarkomen von therapeutischem Nutzen sein kann, wird sich allerdings noch zeigen müssen.

\section{Fazit für die Praxis}

Knorpeltumoren weisen einen reifen angiogenen Phänotyp mit distinkten morphologischen Gefäßmustern auf. Angiogene Parameter wie die Gefäßdichte und -proliferation sind für die Differenzialdiagnose von Knorpeltumoren, speziell für die schwierige Abgrenzung von gut differenzierten Chondrosarkomen gegenüber Enchondromen, nicht geeignet. Auch Expressionsanalysen angiogener Faktoren, wie VEGF-A, Ang-1, Ang-2 und ADAMTS1 sind differenzialdiagnostisch nicht hilfreich. Immerhin korreliert die Gefäßproliferation in Knorpeltumoren mit der VEGF-A-Expression. Im Zellkulturmodell kann die Expression von Angiogenesefaktoren in Chondrosarkomzellen durch Hypoxie und IL-1 $\beta$ beeinflusst werden. Die Regulation der Angiogenese könnte möglicherweise von therapeutischem Nutzen sein.

\section{Korrespondenzadresse}

\section{PD Dr. T. Kalinski}

Institut für Pathologie,

Otto-von-Guericke-Universität

Leipziger Str. 44, 39120 Magdeburg

kalinski@med.ovgu.de

Interessenkonflikt. Der korrespondierende Autor gibt an, dass kein Interessenkonflikt besteht.

\section{Literatur}

1. Ayala G, Liu C, Nicosia R et al (2000) Microvasculature and VEGF expression in cartilaginous tumors. Hum Pathol 31:341-346

2. Bovée JV, Cleton-Jansen AM, Rosenberg C et al (1999) Molecular genetic characterization of both components of a dedifferentiated chondrosarcoma, with implications for its histogenesis. J Pathol 189:454-462

3. Carmeliet $P$ (2005) Angiogenesis in life, disease and medicine. Nature 438:932-936

4. Eberhard A, Kahlert S, Goede V et al (2000) Heterogeneity of angiogenesis and blood vessel maturation in human tumors: implications for antiangiogenic tumor therapies. Cancer Res 60:1388-1393

5. Folkman J (1995) Angiogenesis in cancer, vascular, rheumatoid and other disease. Nat Med 1:27-31

6. Hicklin DJ, Ellis LM (2005) Role of the vascular endothelial growth factor pathway in tumor growth and angiogenesis. J Clin Oncol 23:1011-1027

7. Iruela-Arispe ML, Carpizo D, Luque A (2003) ADAMTS1: a matrix metalloprotease with angioinhibitory properties. Ann NY Acad Sci 995:183-190

8. Jones PF (2003) Not just angiogenesis-wider roles for the angiopoietins. J Pathol 201:515-527

9. Kalinski T, Krueger S, Pelz AF et al (2005) Establishment and characterization of the permanent human cell line C3842 derived from a secondary chondrosarcoma in Ollier's disease. Virchows Arch 446:287-299

10. Kalinski T, Krueger S, Sel S et al (2007) ADAMTS1 is regulated by interleukin-1 beta, not by hypoxia, in chondrosarcoma. Hum Pathol 38:86-94

11. Kalinski T, Krueger S, Sel S et al (2006) Differential expression of VEGF-A and angiopoietins in cartilage tumors and regulation by interleukin-1 1 beta. Cancer 106:2028-2038

12. Kalinski T, Sel S, Kouznetsova I et al (2009) Heterogeneity of angiogenesis and blood vessel maturation in cartilage tumors. Pathol Res Pract 205:339 335

13. Kawai T, Akira S (2007) Signaling to NF-kappaB by Toll-like receptors. Trends Mol Med 13:460-469

14. Keyt BA, Berleau LT, Nguyen HV et al (1996) The carboxyl-terminal domain (111-165) of vascular endothelial growth factor is critical for its mitogenic potency. J Biol Chem 271:7788-7795

15. Lin C, McGough R, Aswad B et al (2004) Hypoxia induces HIF-1alpha and VEGF expression in chondrosarcoma cells and chondrocytes. J Orthop Res 22:1175-1181

16. Ozerdem U, Stallcup WB (2003) Early contribution of pericytes to angiogenic sprouting and tube formation. Angiogenesis 6:241-249

17. Porter S, Clark IM, Kevorkian L, Edwards DR (2005) The ADAMTS metalloproteinases. Biochem J 386:15-27
18. Rodriguez-Manzaneque JC, Westling J, Thai SN et al (2002) ADAMTS1 cleaves aggrecan at multiple sites and is differentially inhibited by metalloproteinase inhibitors. Biochem Biophys Res Commun 293:501-508

19. Schipani E, Ryan HE, Didrickson S et al (2001) Hypoxia in cartilage: HIF-1alpha is essential for chondrocyte growth arrest and survival. Genes Dev 15:2865-2876

20. Tait CR, Jones PF (2004) Angiopoietins in tumours: the angiogenic switch. J Pathol 204:1-10

21. Tanaka F, Ishikawa S, Yanagihara K et al (2002) Expression of angiopoietins and its clinical significance in non-small cell lung cancer. Cancer Res 62:7124-7129

22. Uemura A, Ogawa M, Hirashima M et al (2002) Recombinant angiopoietin-1 restores higher-order architecture of growing blood vessels in mice in the absence of mural cells. J Clin Invest 110:16191628

23. Uthoff SM, Duchrow M, Schmidt MH et al (2002) VEGF isoforms and mutations in human colorectal cancer. Int J Cancer 101:32-36

24. Vaupel P, Harrison L (2004) Tumor hypoxia: causative factors, compensatory mechanisms, and cellular response. Oncologist 9 (Suppl 5):4-9

25. Voronov E, Shouval DS, Krelin Y et al (2003) IL-1 is required for tumor invasiveness and angiogenesis. Proc Natl Acad Sci U S A 100:2645-2650

26. Yancopoulos GD, Davis S, Gale NW et al (2000) Vascular-specific growth factors and blood vessel formation. Nature 407:242-248

27. Yonenaga $\mathrm{Y}$, Mori A, Onodera $\mathrm{H}$ et al (2005) Absence of smooth muscle actin-positive pericyte coverage of tumor vessels correlates with hematogenous metastasis and prognosis of colorectal cancer patients. Oncology 69:159-166 